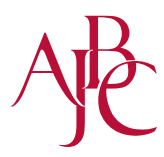

\title{
The Issues of Redundant Publication Based on Some Questions
}

In-Jae Lee

Department of Ethics Education, Seoul National University of Education, Seoul, Korea

Corresponding author: In-Jae Lee, Department of Ethics Education, Seoul National University of Education, 96 SeochoJoongang-ro, Seocho-gu, Seoul 06639, Korea

Tel.: +82 234752411

Fax: +82 234752032

Email: ijlee@snue.ac.kr

Received May 26, 2016

Revised June 22, 2016

Accepted June 23, 2016

Published June 30, 2016

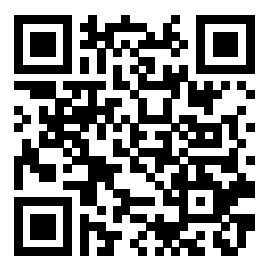

\begin{abstract}
Purpose: This paper analyses the issues of redundant publication which have been the hot topic in publication ethics and suggests a desirable publication practices which researchers should understand and comply with. Methods: I have collected the hot questions from audiences and researchers who would attend on my research ethics lecture and respond them. Results: It was identified meaning and differences in similar terms related redundant publication and was trying to solve the questions and misunderstandings of researchers by describing the main 10 questions of redundant publication with specific examples. Conclusion: Researcher should present the sources adequately and clearly when they use their previously published papers. It is recognized that when researchers publish their paper whose content was same or substantially similar to that of a previously published one without adequate cross-referencing and then take advantage of it unduly, that is unjustifiable redundant publication.
\end{abstract}

Keywords: Redundant publication, Duplicate publication, Self plagiarism, Publication ethics

\section{Introduction}

우리는 연구자가 연구 성과를 발표 및 게재하는 과정에서 잘 모 르고 한 것이든 의도적으로 한 것이든 연구윤리를 위반하는 대 표적인 것으로 표절과 함께 중복게재(redundant publication) 가 있다는 것을 알고 있다. 연구윤리의 중요한 하위 영역으로 서 출판윤리(publication ethics)가 있는데, 출판윤리에서는 표절과 함께 중복게재 및 저자 자격(authorship)에 관한 쟁점 이 핵심적으로 논의되곤 한다. 연구자들 중에는 표절과 함께 중 복게재에 대해 많이 궁금해 하는 사람들이 많다. 특히 중복게 재의 의미나 그것이 왜 문제가 되는지에 대해서는 어느 정도 알 고 있지만 구체적으로 어떤 때에 중복게재에 해당되는지에 대 해서는 쉽게 판단할 수 없다고 말한다. 그만큼 중복게재를 둘 러싼 논의에서 학문 분야, 연구의 방법이나 특성에 따라 중복 게재를 판단하는 요소나 기준에서 차이가 크기 때문이다. 따라 서 본 논문에서는 중복게재를 둘러싼 여러 쟁점과 관련하여 연 구자들이 보다 명확하게 알고 싶어 하고 또 구체적인 설명이 필요하다고 느끼고 있는 질문들을 모아 이에 답하고자 한다.
이를 위해 필자는 평소 대학의 교수나 대학원생 그리고 연구소 의 연구자를 대상으로 연구윤리 강의를 했을 때 나왔던 질문들 과 이메일이나 학술 행사 등에서 개인적으로 받았던 질문들을 몇 가지로 유형화하여 이에 대한 필자의 견해를 밝히는 방식으로 기술하였다.

\section{The main issues of redundant publication}

\section{1. 중복게재, 자기표절, 이중게재라는 용어가 함께 사용되고 있는데 구체적으로 어떻게 다른가요?}

중복게재는 표절과 함께 연구윤리의 주요 영역 중의 하나인 출판윤리(publication ethics)의 중심 주제이다. 표절이 연구 자가 타인의 독창적인 아이디어나 연구 결과를 적절하게 출처 를 표시하지 않고 마치 자신의 것처럼 활용하여 생기는 윤리 적, 법적 문제와 관련된다면, 중복게재는 연구자 자신의 이전 연구를 이후의 연구에 활용할 때 분명하게 출처를 표시하지 않 고 마치 처음으로 발표(게재)하는 것처럼 하여 생기는 윤리적, 
저작권 침해의 문제와 관련된다. 자신이 이미 발표(게재)한 연구 결과를 적절하게 출처를 밝히지 않고 다시 사용하는 것 (reuse)과 관련하여 사람마다 선호하는 용어가 다르고 또 그 사 용 빈도에서 다소 차이가 있지만, 유사한 현상에 대하여 다른 용 어가 혼용되고 있다. 그 대표적인 용어에는 중복게재(redundant publication), 이중게재(duplicate publication), 텍스트 재활 용(text recycling), 논문 쪼개기(salami slicing)가 있다.

중복게재란 원저자에 의해 이미 게재된 내용의 일부 또는 모 든 것이 적절하게 출처표시 없이 반복되어 사용되는 출판의 한 형태를 의미한다. 다시 말하면, 연구자 자신의 최초의 저작물 이 게재되었다는 점을 밝히지 않고 동일한 또는 실질적으로 유 사한 내용을 동일 언어 또는 다른 언어로 한 번 이상 출판하는 것을 말한다. 이중게재는 중복게재와 동의어로써 중복게재보다 는 더 느슨하게 사용되기도 하지만, 엄격하게 말하면 이미 게 재된 논문과 동일한 것을 두 번째로 게재하는 것을 말한다. 텍 스트 재활용은 저자들이 한 번 게재한 것보다 더 많이 텍스트 를 다시 사용하는 것을 말한다. 표준화된 연구 방법의 반복이나 공통적인 데이터의 출처를 기술할 때처럼 어떤 경우에는 이러 한 텍스트 재활용은 정당화될 수도 있다. 만일 저자가 몇 개의 chapter, editorial, 유사한 주제에 대한 주석(commentaries) 을 써야 할 때 일정 부분 텍스트의 재활용은 불가피할 수가 있 다. 그러나 저작권은 항상 존중되어야 하므로 저자는 출판사 편 집인에게 유사한 자료가 이미 게재되었다는 점을 알려야 한다. 대부분의 사람들은 텍스트의 재활용을 데이터의 반복 사용 보 다는 문제가 크지 않다고 간주하기도 한다. 논문쪼개기는 하나 의 연구(a single study)에서 나온 데이터를 몇 개의 연구로 나 누어 출판하는 관행을 일컫는 데, 가장 분명한 방식으로 연구를 제시하는 것보다는 저자의 업적을 부풀리기 위한 것으로 사용 되기 때문에 부적절한 것이다(Wager, 2015).

다른 한편, 미국의 대표적인 출판윤리의 전문가인 로이 그(M. Roig)에 의하면, 자기표절이라는 포괄적 개념 속에 (1) 중복게재(독자나 출판사에 알리지 않고 이미 출판된 것 과 동일한 논문을 다시 출판하는 것), (2) 쪼개기 출판(salami publication, fragmented publication), 또는 하나의 논문을 여러 개로 나누는 것, (3) 텍스트 재사용(text recycling), (4) 저 작권 침해(copyright infringement) 등 4가지로 구분함으로써 중복게재를 자기표절의 한 형태로 규정하고 있다(Roig, 2009; Lee, 2015).

\section{2. 중복게재가 문제가 있다면, 새로운 논문을 쓸 때 자신의 이 전 연구 성과를 활용해서는 안 된다는 것인가요?}

학술적 연구가 매번 새로운 주제로만 이루어지는 것만 아니 라, 선행 연구의 중요한 아이디어나 성과를 바탕으로 심화, 확 대되는 특성도 지니기 때문에 연구자 자신의 이전 연구 결과를
이후의 연구에서 활용하는 것 자체를 금지하는 것이 아니다. 문 제는 이미 발표(게재)된 자신의 연구 내용을 다시 활용하면서도 명확하게 출처를 밝히지 않아 마치 처음 발표(게재)하는 것처럼 속인다는 데 있다. 매우 심각한 것은 이미 게재된 논문과 동일 또는 실질적으로 유사한 논문을 출처를 밝히지 않고 다시 게재 하여 별도의 연구 업적으로 인정을 받는 것이다.

학술 연구에서 자신의 이전 연구 결과를 활용할 때 지켜야 할 연구윤리와 관련하여 대체로 국내의 연구자들은 자신의 이 전 저작물을 이후의 저작물에서 활용할 때 출처표시를 하지 않 아도 되는 것으로 인식하고 있는데, 이는 잘못이다. 일반적으 로 거의 대부분의 국내 - 외 학술지들은 그 어디에도 이미 게재 되지 않은 최초의 논문을 출판하는 것을 출판 정책(publication policy)의 기본으로 규정하고 있다. 따라서 연구자는 논문을 투 고할 때, 자신의 이전 연구와 중복되는 것이 있으면 정확하게 출처를 표시하고 이러한 중복성에 관한 정보를 관련 자료와 함 께 학술지 편집인에게 알림으로써 중복게재 여부에 대한 판단 을 받는 것이 바람직하다. 만약 이미 게재된 내용의 일부든 거 의 대부분이든 출처표시를 하지 않고 활용하게 되면, 독자나 업 적평가 기관이 마치 새로운 저작물인 것처럼 오해할 수 있게 만 들기 때문에 문제가 된다.

연구자가 최초의 연구 자료(original data)를 이미 게재한 학 술지 이외에 다른 학술지에 다시 게재하고자 할 때, 이전에 이 미 게재한 학술지에 대해 출처를 밝히지 않는 것은 비윤리적인 것이다. 이는 바로 자신과 타인을 속이는 비윤리적인 행위일 뿐 만 아니라 저작권을 침해할 수도 있기 때문이다. 그러므로 연구 자는 논문을 투고할 때 중복게재를 피하기 위해 세심하게 주의 를 기울어야 한다. 즉, 자신이 이미 게재한 연구 내용을 활용했 으면 반드시 출처를 밝혀야 하며, 자신이 쓴 논문이 중복게재인 지 아닌지를 스스로 검토한 후 문제가 없다고 판단될 때 논문을 투고해야 한다. 특히 중복성을 가진 이전 연구와 비교할 때 명 백하게 학술적 가치 면에서 차이가 날 수 있도록 해야 한다.

\section{3. 중복게재는 항상 비난을 받거나 허용할 수 없는가요?}

중복게재가 꼭 비난을 받거나 허용할 수 없는 경우만 있는 것이 아니다. 다시 말하면, 허용할 수 없고, 비난의 대상이 되 는 중복게재도 있지만, 허용할 수 있는 중복게재도 있기 때문 이다. 먼저 비난의 대상이 되거나 허용할 수 없는 중복게재에 어떤 것이 있는지를 살펴보자. 2015년 11월 3일에 교육부 훈 령 제 153 호로 제정된 〈연구윤리확보를 위한 지침〉을 보면, "부 당한 중복게재”를 연구부정행위의 한 유형으로 제시하고 있 다. 이에 의하면, 부당한 중복게재란 "연구자가 자신의 이전 연 구결과와 동일 또는 실질적으로 유사한 저작물을 출처표시 없 이 게재한 후, 연구비를 수령하거나 별도의 연구 업적으로 인 정받는 경우 등 부당한 이익을 얻는 행위”이다. 즉, 이는 학문 
분야를 막론하고 허용할 수 없는 최소한의 기준으로 이중게재 (duplicate publication) 된 논문을 활용하여 부당한 이익을 얻 은 경우를 제시한 것으로 이해할 수 있다. 이 외에도 연구부정 행위로 명확하게 규정되지 않았지만, 연구자 자신의 이전 연구 의 일부분을 적절하게 출처를 밝히지 않고 다시 활용함으로써 마치 처음 사용하는 것처럼 오해케 하는 것은 금지되고 있는 출 판행위이다.

다음으로 허용할 수 있는 중복게재의 유형에는 이차게재 (secondary publication)가 있다. 이는 자신의 학술적 저작물 을 널리 알리기 위해 먼저 게재된 학술지와 후에 게재될 학술 지 모두의 동의를 받아 출처를 밝히고 게재한 경우를 말한다. 이차게재는 언어를 달리하여 게재하는 경우가 많은데, 이와 같 이 다른 언어로 번역한 번역저작물은 저작권법에서 2 차적 저작 물로 별도의 저작권을 갖게 되고, 자신의 논문을 다른 언어로 번역하여 해당 학술지에 싣는 것은 연구 성과의 확산에 기여할 뿐만 아니라 동일한 독자가 중복게재에 의해 피해를 입을 가능 성이 적다는 의미에서 중복게재에 포함되지 않는 것으로 보아야 한다(Lee, 2015).

\section{4. 중복게재의 한 유형으로서 살라미 논문(salami slicing)이란 구체적으로 무엇인가요?}

살라미 논문의 정확한 의미에 관하여 연구자 간, 학문 분야 별 간 여전히 많은 논란이 있다. 어떤 사람들은 살라미 논문을 중복되는 자료들을 만들어 내는 것, 즉 재출판(republication) 의 다른 형태로서 대체로 동일한 가설, 방법론, 결과를 공유하 는 것이라고 생각한다. 재출판(republication)이란 이미 출판된 것과 실질적으로 중첩되는 논문을 이전 출판에 대해 명백하게, 가시적으로 언급하지 않고 출판하는 것으로 대표적으로 첫째, 명백한 이중게재로 동일한 자료, 방법, 결론을 가진 연구와 둘 째, 의심되는 또는 잠재적으로 이중게재라고 여겨지는 것으로 거의 동일한 자료, 방법, 결론을 갖는 연구가 포함된다(Wong, 1990). 그렇지만 살라미 논문은 샘플이 같고, 연구 결과나 텍스 트는 다르고, 두 논문의 언어는 같거나 다를 수도 있다. 다른 사 람들은 “최소한의 출판 단위(least publishable units)" 라는 용 어를 사용하는데, 단일의 논문으로 출판하는 것이 횔씬 더 적합할 수 있는 자료로부터 여러 개의 논문을 출판하는 것을 말한다. 그렇 지만 어떤 용어를 사용하든 살라미 논문은 부적절하고 윤리적이지 않는 것으로 간주되고 있다.

그러나 다른 한편, 살라미 논문을 정당한 관행이 될 수 있다 고 생각하는 사람들도 있다. 여기에 해당될 수 있는 전형적인 사례는 다수의 목적과 분석(multiple objectives and analyses) 을 가진 대규모 연구일 때이다. 다른 말로 말하면, 만일 단일의 샘플/코호트 또는 data set이 다른 결과를 가진 다른 가설을 검 증하기 위해 사용되었다면, 또는 동일하거나 유사한 source로
부터 나온 데이터를 분리하는 것이 더 적절하다면 살라미 논문 은 인정받을 수 있다. 이것은 동료 연구자나 독자들에게 훨씬 더 제시할만하고 읽을 가치가 있어 유익함을 주기 때문이다.

살라미 논문이 항상 비윤리적인 것이 아니라고 할지라도, 그 것은 많은 사람들에 의해 속이는 행위로 간주되곤 한다. 그러므 로 최소한 이전에 출판된 논문은 후속하는 논문에서 반드시 출 처를 밝혀야 한다. 뿐만 아니라 저자들은 처음 출판한 논문에서 가능하다면 후속 연구를 수행하고 이를 출판하고자 한다는 점 을 밝히는 것이 바람직하다. 살라미 논문은 횔씬 더 길게 후속 데이터를 보고하지만 방법, 결과 그리고 결론이 index 논문과 동일하거나 매우 유사하기 때문이다(Adibi et al., 2015).

\section{5. 중복게재를 판단하는 기준은 무엇인가요?}

중복게재란 자신의 이전 연구 내용과 동일 내지 실질적으로 유사한 것을 그 원출처를 밝히지 않고 같은 언어로 또는 다른 언어로 한 번 이상 출판하는 것을 말한다. 여기서 비교되는 두 개의 논문이 반드시 동일해야만 중복게재인 것은 아니다. 데이 터가 같거나 실질적으로 겹친다거나 유사한 결과를 제시하면 서도 그것에 대해 적절하게 출처를 표시하지 않으면 중복게재 라고 할 수 있다. 엄밀하게 말하면, 여기서 중복되었다는 말은 전자 출판이든 인쇄된 것이든, 동일한 언어든 다른 언어든, 완 전히 같을 수도 있고 부분적으로 겹칠 수도 있다는 것으로 해 석될 수 있다. 여기에는 새로운 데이터가 포함될 수도 있고, 원 래 출판된 것의 결과를 단지 복제하기도 하지만 이에 대한 출 처를 밝히지 않는 것을 말한다.

중복게재인지 아닌지를 판단할 때 어떤 기준을 활용하는가? 물론 중복게재를 판단하는 기준은 학문 분야나 학술지마다 다 를 수 있지만, 이전에 발표 내지 게재되지 않은 최초의 논문을 투고해야 한다는 학술지의 출판 규정을 고려할 때, 가장 기본 적이고도 일반적인 중복게재의 판단 기준은 이미 게재된 내용 을 적절하게 출처를 밝히지 않고 다시 활용하는 것이다.

중복게재를 판단하는 기준으로 우리가 참조해 볼 만한 것으 로는 흥부외과학 저명 학술지 편집인들의 모임에서 제시한 다 음과 같은 6 가지 항목이다. 이를 자세히 살펴보면 다음과 같다 (Lee, 2015). 첫째, 가설이 유사하다(가설 중 인구집단 관련 독 립변수와 종속변수가 거의 동일하다), 둘째, 표본의 수나 크기 가 유사하다(연구 재료, 실험동물, 연구 대상자의 $90 \%$ 이상이 거의 유사하다), 셋째, 방법이 동일하거나 유사하다(자료 수집, 분석, 제시 방법이 같거나 거의 유사하다), 넷째, 결과가 유사 하다(결과가 양이나 질 측면에서 거의 동일하다), 다섯째, 최소 한 저자 1 명이 공통적이다(제 1 , 제 2 , 마지막 저자가 PubMed 에 이름이 있는 경우), 여섯째, 새로운 정보가 없거나 있다고 해도 매우 적다(추가적인 지식이 거의 없는 경우). 이 이외에도 중복게재를 판단하는 데 있어 활용되는 기준으로는 비교되는 
두 저작물 사이에 서로 출처(cross reference)를 언급하지 않으 며, 참고문헌도 매우 유사하다는 점을 들 수 있다.

6. 이미 단행본의 한 chapter로 사용된 글을 다른 책에서 또 묶어 총서로 낼 때, 즉, 재사용하는 것이 중복게재 또는 자기표 절에 해당하는가요?

자신의 이전 저작물을 다시 사용하는 것 자체가 자기표절 또 는 중복게재인 것은 아니다. 이전에 발표 내지 게재된 자신의 저작물을 다시 활용하면서도 적절하게 출처표시를 하지 않아 마치 새로운 것을 처음 발표 내지 게재한 것처럼 하였을 때 연 구윤리에 어긋난다. 이미 출판된 단행본의 한 챕터의 내용을 다 시 재활용하고자 할 때에는 해당 출판사의 허락을 받아(또는 최 소한 출처표시를 하고), 활용하여야 자기표절 또는 저작권 침해 의 소지를 예방할 수 있다. 처음 출판된 책의 내용을 그대로 활 용하지 않고 보완 및 새로운 내용이 추가되었다고 하더라도 이 미 발표한 것과 겹치는 부분에 대해서는 출처표시를 정확하게 해야 한다. 만약 어느 학회나 출판사에서 이미 출판된 단행본의 한 챕터의 내용을 다시 사용할 수 있도록 요청을 해 왔다면, 이 미 출판된 책의 출판사에게도 알려 허락을 구하고, 출처표시를 하면서 재활용하는 것이 바람직하다. 또한 재활용된 저작물에 대한 업적을 인정받는데 있어 유의해야 할 사항은 앞서 출판한 것과 내용이 동일 또는 실질적으로 유사하다면, 업적 인정을 부 당하게 두 번 받지 않도록 해야 한다.

7. 국가 R\&D 연구사업의 지원을 받아서 작성한 결과를 논문으 로 학회지에 게재하였고, 이것을 활용하여 학위논문을 작성하 려고 합니다. 이때, 저자표시는 어떻게 하는 것이 적절한 방법 인가요? 연구비를 지원받아 수행한 연구결과를 활용해서 학위 논문으로 다시 작성하면 중복게재에 해당되는가요?

위의 물음은 다음과 같이 두 가지 경우로 나누어서 생각해 볼 수 있다. 하나는 개인이 단독으로 국가 R\&D 연구 사업의 지원 을 받아 수행했을 때이고, 다른 하나는 공동으로 연구를 수행한 경우이다.

먼저 전자의 경우 저자표시를 어떻게 해야 바람직한지를 살펴 보자. 한 연구자가 단독으로 연구비를 지원 받아 연구를 수행하 고, 그 결과를 학술지에 게재하였다. 다시 이것을 활용하여 학위 논문을 작성하고자 할 때, 이미 게재된 내용(중요한 아이디어나 연구자의 고유한 핵심 단어, 문장, 표, 그림, 그래프, 사진 등의 텍스트)을 학위논문에서 재활용할 때는 반드시 출처를 명확하게 밝혀야 한다. 이때 학위논문의 저자는 당연히 연구자 개인이 된다.

다음으로 후자의 경우, 즉 여러 사람이 공동으로 연구를 수행 하고, 그 결과를 학술지에 공동으로 게재하고, 다시 여기에 있는 내용을 활용하여 학위논문을 작성한 경우, 앞에서 설명한 것과 마찬가지로 활용한 내용에 대해서는 출처표시를 정확하게 하여
야 한다. 물론 이 학위논문의 저자는 바로 연구자 자신이 된다. 그런데 여기서 주의해야 할 것은 활용한 연구 내용이 공동 연구 자의 공동 작업의 결과이므로 이를 활용하고자 할 때에는 공동 저자들에게 충분히 알리고 동의를 받아야 한다.

한 연구자가 연구비를 지원받아 수행한 연구결과를 활용해서 자신의 학위논문으로 다시 작성하고자 할 때, 먼저 연구비 지원 기관에 제출한 연구결과 보고서가 공식 간행된 것이 아니라고 인정받는 경우, 출처표시 없이 사용하여도 중복게재에 해당되 지 않는다. 그렇지만, 제출한 연구결과 보고서가 연구비 지원기 관에서 공식적으로 발간되어 널리 인지되는 것이라면, 이를 학 위논문에서 활용할 때는 반드시 정확하게 출처를 표시해야 중 복게재의 해당되지 않는다.

이 경우 연구자가 특히 유의해야 할 사항이 있다. 그것은 바로 연구비 지원 기관과 연구 협약을 할 때 연구결과의 사용에 대하 여 맺은 약정을 확인하고 그에 따라야 한다는 것이다. 일반적으 로 연구 결과물은 연구비 지원기관에 귀속하므로, 이 연구 결과 물을 활용하여 학술지에 논문으로 게재하고자 할 경우에는 협약 사항이 정한 바를 따르거나 연구비 지원 기관으로부터 허락을 받 고 논문을 게재하여야 한다. 물론 게재된 논문에 대해서는 어느 기관으로부터 연구비를 지원받았다는 사사표시를 하여야 한다.

8. 학위논문을 학술지에 투고하여 게재할 때, 지도교수가 아닌 사람이 공동저자로 등록되는 것이 연구윤리에 위배되는가요? 이과 계열의 공동연구에서 연구에 기여한 부분이 있다면, 학위 논문을 발췌하여 학회지에 투고할 때, 지도교수가 아니더라도 공동저자로 등록하는데 전혀 문제가 되지 않는다고 생각합니 다. 현재 이를 금지하고 있는 규정은 없는 것으로 알고 있으며, 오히려 연구에 참여했음에도 지도교수가 아니기 때문에 공동 연구의 저자에서 배제한다면, 이는 연구에 참여하여 기여한 사 람에게 저자의 자격을 배제하는 것으로 '교육부 연구윤리 지침 제12조'에 위배될 수 있다고 생각합니다. 그리고 교육부나 한 국연구재단에서 발주한 과제를 진행하는 경우에도 공동연구를 수행하는 경우, 연구에 참여한 대학원생이 학위논문을 작성한 후, 그 논문을 발췌하여 학회지에 투고하는 경우가 많습니다. 이때에도 연구에 참여한 공동연구원들을 공동저자로 등록하는 것이 당연시되고 그 사례도 매우 많습니다.

학위논문을 학술지 논문으로 게재할 경우, 〈연구윤리 확보 를 위한 지침(교육부 훈령, 2015.11.3.)〉은 "지도교수가 단독 으로, 또는 학위논문의 저자가 아닌 제 3 자가 제 1 저자(주저자) 로 학술지 등에 게재/발표하는 경우”를 연구부정행위 범주에 포함시키고 있다. 이는 기본적으로 학위논문과 거의 같거나 실 질적으로 같은 내용을 요약 내지 발췌하여 학술지 논문으로 전 환하여 게재할 경우, 해당 논문에서 실질적으로 주요한 역할을 한 사람이 학위논문의 저자로 보기 때문에 그에게 제 1 저자(주 
저자)로서의 업적을 배분하는 것이 타당하다고 보기 때문이다. 물론 학위논문에 기반을 두었지만 학위논문과 학술적으로 의미 있는 차이가 있는 내용을 포함한 학술지 논문이 게재되었고, 이 과정에서 지도교수 또는 제 3 자가 그 의미있는 차이에 대하여 실질적 역할을 하였고, 그것이 제 1 저자로서의 역할이었다면 학 술지 논문에서 이에 부합한 저자로 등재되는 것은 타당하다고 본다. 여기서 중요한 것은 학위논문을 발췌하여 학술지에 논문 을 투고할 때 저자로서의 역할을 실질적으로 하지 않은 사람에 게 저자의 자격을 주거나 저자로서의 정당한 역할을 했음에도 저자에서 배제하는 것은 연구부정행위에 속한다는 것이다. 학 위논문의 내용을 거의 그대로 또는 단순히 요약 내지 발췌하여 학술지에 게재한 경우라면, 학위논문의 저자나 지도교수가 각 각 제 1 저자 그리고 공동저자로 등재하는 것이 바람직하고, 그 이외의 경우, 즉 제 3 자가 제 1 저자 또는 공동 저자로 등재되는 것은 이에 합당한 역할을 실질적으로 하였다고 볼 수 있는 타당 한 근거가 없기 때문에 연구윤리에 위반된다고 할 수 있다.

학위논문의 내용을 활용하여 학술지 논문으로 게재할 때, 학 위논문 심사위원으로 참여한 외부교수가 교신저자가 되는 경우 에 이는 연구윤리에 어긋나지 않는 것인가? 이 경우도 위에서 논의한 원리를 적용하면 어렵지 않게 판단할 수 있다고 본다. 즉, 학위논문의 내용과 동일 내지 실질적으로 유사한 내용을 학 술지에 게재한 것이라면, 외부 심사 위원이 교신저자로 등재되 는 것이 정당하다고 판단할 수 있는 합리적인 근거가 충분하지 않다. 그러나 학위논문을 토대로 출발하였지만 학위논문의 내 용과 다른 학술적 가치를 포함한 내용이 추가되었고, 이 부분을 외부 심사 위원이 기여하였고, 이것이 교신저자로서의 역할에 부합된다고 인정될 때에는 교신저자로 등재되는 것이 연구윤리 적으로 문제가 되지 않는다고 본다.

\section{9. $A$ 교수는 교내 연구비를 신청하여 선정된 후, 대학원생 $B$ 와 공동연구를 수행하였습니다. 그 결과물을 이용하여 $\mathrm{B}$ 는 석사 학위 논문을 학교에 제출하였고, 그 후 $\mathrm{A}$ 와 $\mathrm{B}$ 는 $\mathrm{B}$ 의 석사학위 논문을 발췌하여 공동 저자로 학회지에 논문을 게재하였습니 다. 이 경우 A교수의 행위는 연구윤리에 위배되나요?}

교내 연구비를 지원받아 대학원생과 공동연구를 한 후, 이를 학술지에 공동저자로 게재한 것은 정상적인 학술활동이라고 할 수 있다. 다만 이 경우와 관련하여 생각해 보아야 할 것은 교내 연구비 지원의 결과로 나오는 연구물은 통상 이미 나와 있는 연 구 성과와 비교하여 학술적 가치를 갖고 있으면서 독자적으로 도(별도의) 업적으로 인정을 받을 수 있는 정도의 의미있는 차 이가 있어야 한다는 것이다.

이 사례의 경우, 공동 연구자가 석사과정에 있는 대학원생이 었고, 연구 결과물이 먼저 석사학위 논문으로 제출되었으며, 이 를 발췌하여 공동 저자로 학술지에 게재하였으므로, 외면상으
로 보면 대학원생의 석사학위 논문을 발췌하여 학술지에 게재 하고 이를 연구비 지원에 대한 결과로 제출한 것이 된다. 그런 데 석사학위 논문을 거의 그대로 발췌한 것이므로 석사학위 논 문과 실질적인 차이가 없다. 만일 석사학위 논문과 학술지 게재 논문을 비교하여 차이가 있는 부분이 있으면서 이것이 학술적 가치를 갖고 또한 독립적인 연구 논문으로서의 의미 있는 차이 가 있다면, 그리고 석사학위 내용과 겹치는 부분은 적절히 인용 내지 출처를 밝히고, 차이가 나는 부분을 $\mathrm{A}$ 교수가 실질적으로 하였다면, A교수는 연구윤리를 위배하였다고 말할 수 없다.

만일 학위논문이 공식적으로 출판되지 않은 미간행물로 간 주한다고 하더라도 통상 학위논문의 저자는 제자인 B이므로 이 경우는 $\mathrm{A}$ 교수가 $\mathrm{B}$ 와 공동으로 연구를 수행하였다고 하더라도 이미 하나의 업적(학위논문)으로 나온 것을 $\mathrm{A}$ 의 업적으로 하여 연구비 수행 결과로 제출한 것은 사려깊지 못했다고 본다.

10. A교수는 대학원생 C와 공동연구를 수행하였고, 이를 토대 로 $\mathrm{C}$ 는 석사학위 논문을 학교에 제출하였습니다. 그 후 $\mathrm{A}$ 와 $\mathrm{C}$ 는 학회지에 석사학위 논문을 발췌하여 공동저자로 논문을 게 재하였고, 그 결과물을 학교에 제출하여 이 연구 성과에 따른 연구비를 수령하였습니다. 이 경우 A교수의 행위는 연구윤리 에 위배되나요?

이 사안도 앞서 살펴 본 7 의 경우에 유사하다고 볼 수 있다. 결론적으로 말하면, 교내든 교외든 연구비를 받아 수행된 연구 결과물은 통상 이전에 발표(연구)되지 않은 내용을 담고 있기를 연구비 지원 기관의 입장에서는 기대하기 때문에 이미 발표 또 는 게재된 내용을 연구 결과물로 제출하는 것은 이러한 기대를 위배하는 것이므로 바람직하지 않다.

물론 이러한 것과 관련하여 연구비 지원 기관에서 별도로 정 한 규정이 있다면, 즉, 이를테면, "학위논문으로 먼저 제출하고 이를 발췌하여 학술지에 게재한 경우에도 연구비 지원에 따른 실적물로 인정한다."라는 규정이 있다면 예외일 수 있다고 본 다. 아무튼 대학원생과 공동으로 연구를 수행한 후, 먼저 석사 학위 논문으로 제출하고, 다시 이를 발췌하여 전문 학술지에 공 동으로 게재하는 경우, 공동 저자로서의 업적 인정을 받는 데는 큰 문제가 없어 보이지만, 연구비 지원 결과로서 결과물 제출과 관련해서는 오해를 받을 소지가 있다. 이를테면, 석사학위 논문 을 활용하여 연구비 지원을 받았다는 점이다. 내용상으로는 석 사과정 학생과의 공동연구를 하는 경우, 이해가 되는 부분이 있 지만, 연구비를 수혜받고자 할 때에는 보다 엄격하게 구분하여 접근하는 것이 필요하다. 


\section{Conclusion}

지금까지 중복게재와 관련하여 연구 현장에서 궁금해 하는 내 용을 질문으로 구성하고 필자의 의견을 답변으로 제시하였다. 학 술적 글쓰기와 출판 분야에서 널리 적용되고 있는 잉겔핑거 규칙 (the Ingelfinger rule)이라는 것이 있다. 이는 다른 학술지든 매 체든 어딘가(elsewhere)에 이미 출판된 연구 결과를 다시 출판 하지 않는다는 것으로 1969년 잉겔핑거(Franz J. Ingelfinger)가 처음으로 주창한 이래 수많은 학술지에서 이 규칙을 따르고 있다 (Angell \& Kassirer, 1991). 이 규칙은 후에 한 논문이 동료심사 를 거쳐 출판되기까지 지켜야 할 몇 가지 원칙을 세우는데 중요 한 역할을 하였다. 이를테면, 연구 성과를 공표하기 전에 먼저 어 떤 주장이 과학적으로 타당한지를 항상 확인하여야 한다는 점, 한 연구 성과가 학술지에 게재되기 전에 그것을 대중매체에 먼저 발표하는 것을 금지한다는 점, 그리고 연구자가 부당하게 자신의 연구 업적을 부풀리기 위해 중복출판(double publication)을 하지 않아야 한다는 점 등이 바로 그것이다.

연구자는 물론이고 일반 대중들은 학술지에 실린 논문이 뭔 가 새롭고도(fresh) 의미있는 내용을 담고 있을 것이라는 기대 를 갖고 있다. 따라서 이미 어딘가에 게재한 내용을 다시 활용 하면서 적절하게 출처를 밝히지 않아 마치 처음 게재하는 것처 럼 중복게재를 하는 것은 자신은 물론 동료 연구자와 대중을 속 이는 것이다. 이는 연구윤리의 중요한 영역인 출판윤리를 위반 하는 것임은 물론 저작권 침해에 해당될 수도 있다. 따라서 연 구자는 자신의 이전 연구 결과를 활용하여 후속 논문을 작성하 고자 할 때 활용한 부분에 대해 반드시 정확하게 출처를 밝혀야 한다. 특히, 이미 게재한 자신의 연구 결과와 중복되는 내용이 포함된 논문을 작성하여 투고하고자 할 때에는 해당 학술지 편 집자에게 관련 사항에 대하여 충분한 정보를 주고, 출판 여부에 대해 최종 판단을 기다리는 것이 바람직한 연구자의 자세이다.

\section{References}

Adibi P, Kianpour M, Shirani S. Investigating the root causes of duplicate publication in research articles. Journal of Education and Health Promotion, 4: 1418, 2015.

Angell M, Kassirer JP. The Ingelfinger rule revisited. The New England Journal of Medicine, 325: 13711373, 1991.

Lee IJ. Understanding research ethics and ethical practice. Dongmoonsa publishing, Seoul, pp263264, 2015.
Roig M. Avoiding plagiarism, self-plagiarism, and other questionable writing practices: a guide to ethical writing. St. Johns University, 2009.

Wager E. Why is redundant publication a problem? The International Journal of Occupational and Environmental Medicine, 6: 3-6, 2015.

Wong DL. Duplicate publication: a serious concern. Journal of Pediatric Oncology Nursing, 7: 9192, 1990. 


\section{국문초록}

\section{$\mathrm{Q} / \mathrm{A}$ 를 통해 본 중복게재의 쟁점}

이인재

서울교육대학교 윤리교육과, 서울, 한국

목적: 본 논문은 출판윤리의 중요한 영역을 차지하고 있는 중복게재와 관련하여 연구자들이 잘 모르거나 오해하고 있는 점들에 대 해 구체적으로 설명함으로써 연구자가 실천해야 할 바람직한 방안을 탐구하고자 하였다.

방법: 필자가 대학이나 연구 기관의 연구윤리 강의를 통해 얻게 된 중복게재와 관련하여 궁금해 하는 내용을 질문으로 구성하고 이 에 대해 필자가 답변을 하는 형식으로 기술하였다.

결과: 중복게재와 관련된 주요 질문 10 개의 항목에 대해 구체적으로 예를 들어 설명을 함으로써 중복게재와 유사한 용어들의 의미 와 차이점을 규명하였고, 중복게재에 대한 연구자들의 오해와 궁금증이 해소되도록 하였다.

결론: 연구자는 자신의 이전 저작물을 활용하여 후속 논문을 게재할 때 반드시 출처를 정확히 밝혀야 하며, 특히 동일 내지 실질적 으로 유사한 연구를 적절하게 출처를 밝히지 않고 마치 새로운 연구인 것처럼 게재하고 이를 부당하게 활용할 때 '부당한 중복게재' 로서 연구부정행위에 속한다는 점을 인식하여야 한다.

핵심어: 중복게재, 이중게재, 자기표절, 출판윤리

\section{참고문헌}

이인재. 연구윤리의 이해와 실천. 동문사, 서울, pp263-264, 2015. 


\section{中文摘要}

\section{问题解答形式讨论重复发表问题}

李仁宰

首尔教育大学校伦理教育科, 首尔, 韩国

目的: 重复发表占出版伦理的重要领域, 很多研究者对此不太理解或有所误会, 本研究对不理解和误会方面详细说明, 探讨研 究者的正确出版伦理实践方案。

方法: 笔者在大学及研究机关的研究伦理课程中获得相关重复发表的疑惑点, 把这些疑惑点作为问题, 笔者回答的形式阐述了 重复发表问题。

结果: 具体举例说明重复发表相关的十个问题，阐明重复发表类似单词的意义和差异，解答研究者对重复发表的误会和疑惑点。 结论: 研究者利用自己以前的著作物发表后续论文时, 必须正确注明其引用信息, 特别在相同或类似的研究成果中不注明其 引用信息, 好像新的研究成果似的发表或不当利用时, 必须深刻认识到这属于一稿多投重复发表, 研究不道德行为。

关键词: 重复发表，一稿两投，自我剽窃，出版伦理 